\title{
Applicability of viscous hydrodynamics at RHIC
}

\author{
Denes Molnar ${ }^{a, b}$ and Pasi Huovinen ${ }^{a, c}$ \\ ${ }^{a}$ Physics Department, Purdue University, 525 Northwestern Avenue, West Lafayette, IN 47906, USA \\ ${ }^{b}$ RIKEN BNL Research Center, Brookhaven National Laboratory, Upton, NY 11973, USA \\ ${ }^{c}$ Institute für Theoretische Physik, Johann Wolfgang Goethe-Universität, Frankfurt am Main, Germany
}

\begin{abstract}
In an earlier work[1] we established that causal Israel-Stewart viscous hydrodynamics is only accurate in RHIC applications at very low shear viscosities $4 \pi \eta_{s} / s \lesssim 1.5-2$. We show here that the region of applicability is significantly reduced if bulk viscosity plays a role in the dynamics.
\end{abstract}

\section{Introduction}

There has been a lot of recent interest in quantifying the effect of viscosity on observables in heavy-ion collisions at the Relativistic Heavy Ion Collider (RHIC) at Brookhaven. Calculations are most commonly performed using causal dissipative hydrodynamics [2, 3, 4, 5], though covariant transport theory can also be utilized[5, 6, 7].

Hydrodynamics assumes that the system is near local thermal equilibrium. Its region of applicability can only be reliably determined with the help of a fully nonequilibrium theory. In a recent work[1] we used covariant transport to establish the region of validity for the causal Israel-Stewart (IS) formulation of viscous hydrodynamics, in a longitudinally boost invariant setting with a massless $e=3 p$ equation of state (EOS) and only shear viscosity. We found that for typical conditions expected in nuclear collisions at RHIC, IS hydrodynamics is a very good approximation (more precisely, $10 \%$ accurate in computing dissipative effects) when the shear viscosity $\left(\eta_{s}\right)$ to entropy density $(s)$ ratio is not too large, $4 \pi \eta_{s} / s \lesssim 1.5-2$. A useful rule of thumb we obtained is that, in order to reach such accuracy, dissipative corrections to pressure and entropy must not exceed about $20 \%$. This is only a necessary condition but its main advantage is that it can be tested directly from the hydrodynamic calculation.

Here we study the region of validity of IS hydrodynamics for systems with shear and bulk viscosity. Quantifying bulk effects in covariant transport near the hydrodynamic limit is unfortunately unfeasible, at least for a one-component system with $2 \rightarrow 2$ scattering, because bulk viscosity is at least two orders of magnitude smaller than shear viscosity. In order to proceed, we assume that the $20 \%$ rule of thumb above applies in the more general "shear+bulk" case as well.

Because for a massless equation of state bulk viscosity identically vanishes, we here use a more realistic result from lattice QCD[8] (we smoothly merge the EOS onto that of a hadron gas at low $T \lesssim 160 \mathrm{MeV}$ ). Reliable calculations of viscosity in QCD are unfortunately not available for temperatures relevant for RHIC. We therefore focus on the "minimal viscosity" [9] paradigm, i.e., set $\eta_{s}=s /(4 \pi)$. For the bulk viscosity, we consider a Lorentzian in temperature $\zeta=\zeta_{m} s /\left[1+\left(T-T_{c}\right)^{2} / \Delta T^{2}\right]$, where the peak height and width are adjustable parameters. Matching to the bulk viscosity estimate from Meyer[10] gives our default parameterization $\zeta_{m}=$ $0.2, \Delta T=0.03 \mathrm{GeV}$ and $T_{c}=0.192 \mathrm{GeV}$. Though our approach is similar to an earlier study by 
Fries et al[11], the main difference is that we also ensure thermodynamic consistency because we use the complete set of Israel-Stewart equations of motion. We also map out a much wider range of initial conditions.
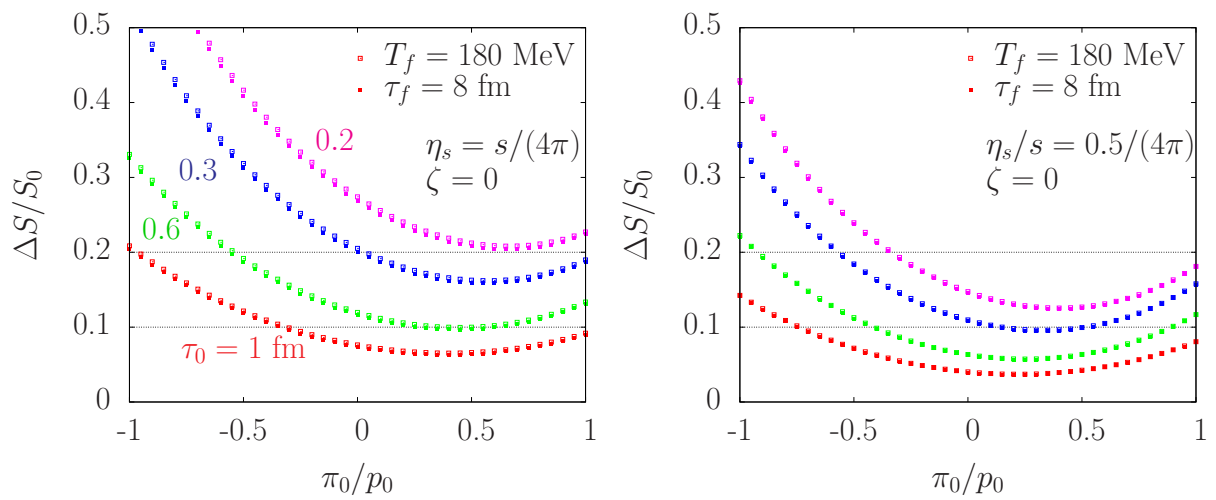

Figure 1: Entropy produced by the time the system cools down to $T=180 \mathrm{MeV}(\tau \approx 8 \mathrm{fm})$ relative to the initial entropy, plotted as a function of thermalization time and initial shear stress, for $\eta_{s} / s=1 /(4 \pi)$ (left) and $0.5 /(4 \pi)$ (right).

\section{Main results}

We solve the complete set of Israel-Stewart equations (cf. [1]) for a boost-invariant Bjorken scenario with axial and transverse translational symmetry. We only highlight here the equation for bulk pressure

$$
\tau_{\Pi} \frac{d \Pi}{d \tau}=-\left(\Pi+\frac{\zeta}{\tau}\right)-\frac{\Pi \tau_{\Pi}}{2}\left(\frac{1}{\tau}+\frac{d}{d \tau} \ln \frac{\zeta}{\tau_{\Pi} T}\right) .
$$

Here $\tau_{\Pi}$ is bulk pressure relaxation time. If the last $\Pi \tau_{\Pi} \ldots$ term is ignored, the entropy generation rate per unit rapidity $d(d S / d \eta) / d \tau=\tau A_{T}\left[\Pi^{2} /(\zeta T)+3 \pi_{L}^{2} /\left(4 \eta_{S} T\right)\right]$ becomes inconsistent with the Israel-Stewart expression for entropy $d S / d \eta=\tau A_{T}\left[s_{e q}-\Pi^{2} \tau_{\Pi} /(2 T \zeta)-3 \pi_{L}^{2} \tau_{\pi} /\left(4 T \eta_{s}\right)\right]\left(\pi_{L}\right.$ is the shear correction to the longitudinal pressure, $\tau_{\pi}$ is the shear stress relaxation time, $A_{T}$ is the transverse area of the system, and $s_{e q}$ is the entropy density in local equilibrium). Therefore, entropy production was overestimated in [11].

Motivated by kinetic theory we set $\tau_{\pi}=6 \eta_{s} /(s T)$, but for simplicity take $\tau_{\Pi}=\tau_{\pi}$ (in kinetic theory $\tau_{\Pi} \approx 5 \tau_{\pi} / 3$ near the massless limit). Our default initial condition for Au+Au at RHIC is $e_{0}=15 \mathrm{GeV} / \mathrm{fm}^{3}\left(T_{0} \approx 0.297 \mathrm{GeV}\right)$ at a thermalization time $\tau_{0}=0.6 \mathrm{fm}$. For $\tau_{0}=0.3$ and $1 \mathrm{fm}$, we scale on an isentropic curve $\tau_{0} s_{0}=$ const. For initial shear stress and bulk pressure we map out wide ranges $-p_{0}<\pi_{0}<p_{0},-p_{0} / 2<\Pi_{0}<p_{0} / 2$ where $p_{0}$ is the initial pressure. Three choices are of special interest: i) local equilibrium (LTE) $\pi_{0}=\Pi_{0}=0$; ii) Navier-Stokes (NS) $\pi_{0}=-(4 / 3) \eta_{s}\left(T_{0}\right) / \tau_{0}, \Pi_{0}=-\zeta\left(T_{0}\right) / \tau_{0}$; and iii) gluon saturation[12] (CGC) $\pi_{0} \approx-p_{0}, \Pi_{0} \approx 0$.

The main quantity we analyze is the entropy produced relative to the initial entropy $\Delta S / S_{0}$. We shall impose $\Delta S / S_{0} \lesssim 0.2$ as the condition for region of validity. Because we only consider longitudinal expansion, we focus on entropy production until the beginning of the hadronic phase $T_{f}=180 \mathrm{MeV}$ (this is almost identical to entropy production until $\tau_{f}=8 \mathrm{fm}$ ). Figure 1(left) shows our results for "minimal" shear and vanishing bulk viscosity. For LTE initial conditions, IS hydrodynamics is applicable when $\tau_{0} \gtrsim 0.3 \mathrm{fm}$. Good accuracy for NS initial conditions 
requires $\tau_{0} \gtrsim 0.6 \mathrm{fm}\left(\pi_{0}^{N S} / p_{0} \approx-0.846,-0.585\right.$, and -0.488 for $\tau_{0}=0.3,0.6$, and $\left.1 \mathrm{fm}\right)$, while for CGC initial conditions even later $\tau_{0} \gtrsim 1 \mathrm{fm}$.
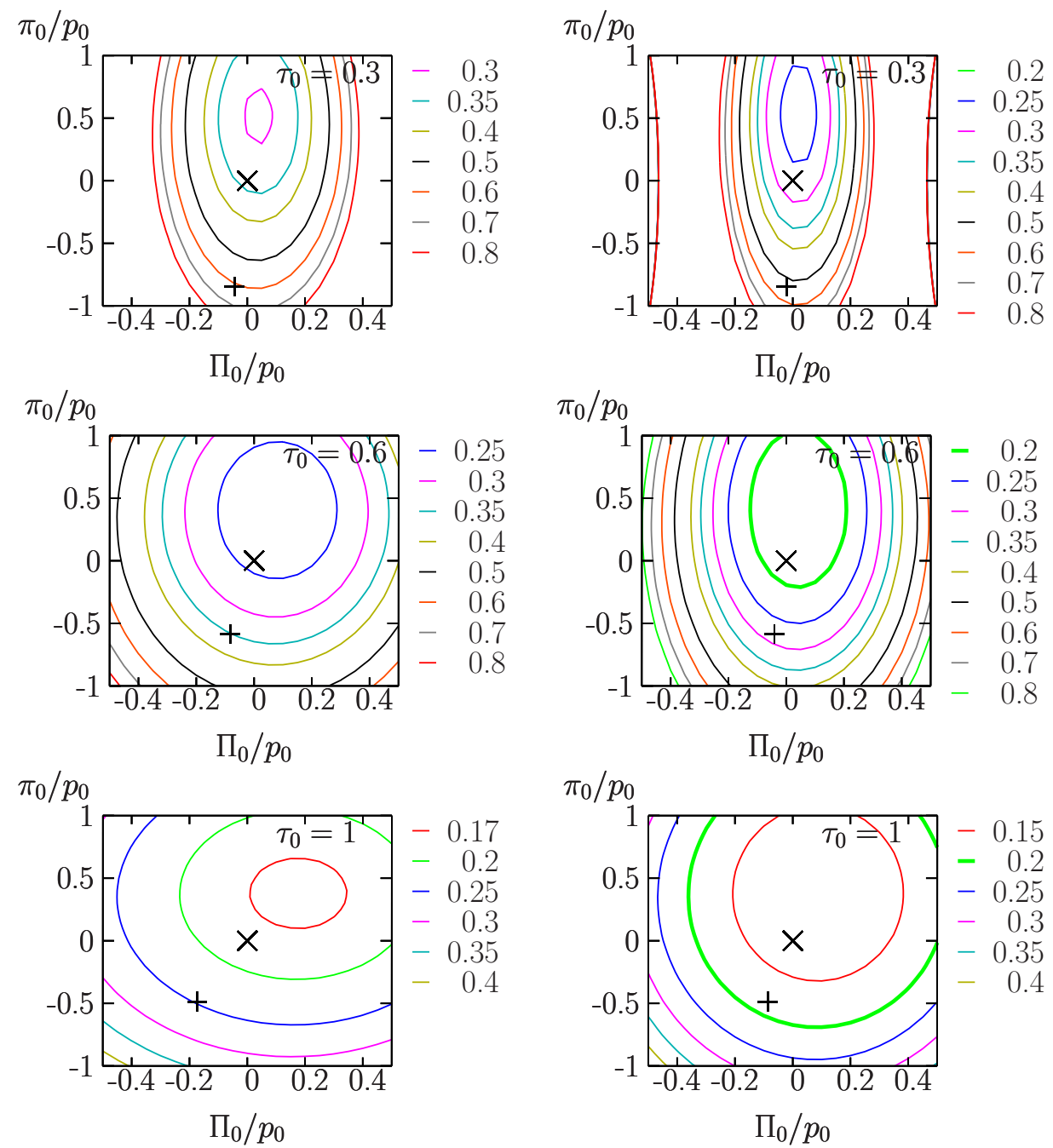

Figure 2: Relative entropy production until $T_{f}=180 \mathrm{MeV}$ as a function of initial shear stress and bulk pressure, for thermalization times $\tau_{0}=0.3$ (top), 0.6 (middle), and $1 \mathrm{fm}$ (bottom). Left column is for $\zeta(T$ ) based on Meyer's calculation[10], right column is for half that large $\zeta(T)$. Crosses indicate local equilibrium initial conditions, while pluses are for Navier-Stokes. In all cases $\eta_{s} / s=1 /(4 \pi)$.

Let us now turn on bulk viscosity. The left column of Figure 2 shows $\Delta S / S_{0}$ for our default $\zeta(T)$ parameterization. Due to the additional entropy produced, LTE initial conditions now require $\tau_{0} \gtrsim 1 \mathrm{fm}$, while simulations from NS and CGC ones necessitate even later thermalization. The situation improves somewhat if bulk viscosity is half as large as our default $\left(\zeta_{m}=0.1\right)$. As seen in the right column, LTE initial conditions are then suitable when $\tau_{0} \gtrsim 0.6 \mathrm{fm}$, while NS can be accommodated if $\tau_{0} \gtrsim 1 \mathrm{fm}$. Results are quite similar if instead the width of the $\zeta(T)$ peak is halved, i.e., $\zeta_{m}=0.2, \Delta T=0.015 \mathrm{GeV}$. We checked that shorter relaxation times help only a 
little, as illustrated in Figure 3 (left) for $\tau_{0}=0.6 \mathrm{fm}$. On the other hand, the region of applicability widens substantially if the shear viscosity is a factor of two smaller (cf. Fig. 3 (right)).
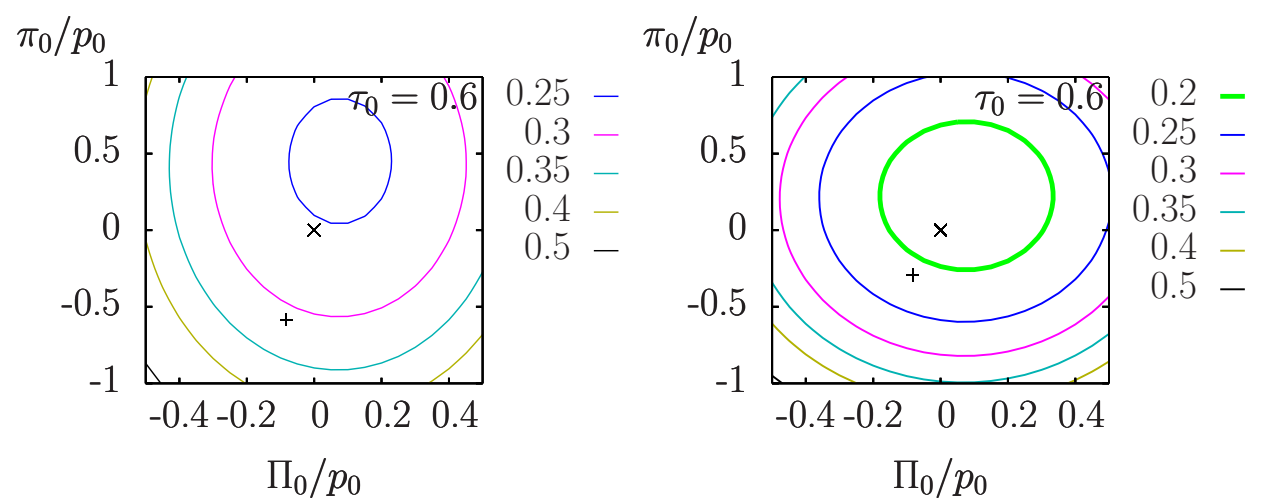

Figure 3: Same as Fig. 2 with our default parameters but halved relaxation times (left), or $\eta_{s} / s=0.5 /(4 \pi)$ (right).

\section{Conclusions}

We study the region of validity of Israel-Stewart viscous hydrodynamics for conditions expected at RHIC, based on the entropy produced during the evolution when both shear and bulk viscosity are present. Our results indicate that, unlike it was previously hoped, viscous hydrodynamics does not extend the range of dynamical description to proper times earlier than $\tau \approx 0.6 \mathrm{fm}$, even for local thermal equilibrium initial conditions - unless the influence of bulk viscosity is basically negligible, or the shear viscosity of hot and dense quark-gluon matter is significantly below the conjectured "minimal" value of $\eta_{s}=s /(4 \pi)$.

\section{Acknowledgments}

We thank RIKEN, Brookhaven National Laboratory and the US Department of Energy [DEAC02-98CH10886] for providing facilities essential for the completion of this work.

\section{References}

[1] P. Huovinen and D. Molnar, Phys. Rev. C 79, 014906 (2009) [arXiv:0808.0953 [nucl-th]].

[2] P Romatschke U Romatschke, Phys. Rev. Lett. 99, 172301 (2007)

[3] H Song and U W Heinz, Phys. Lett. B 658, 279 (2008)

[4] K Dusling and D Teaney, Phys. Rev. C 77, 034905 (2008)

[5] D. Molnar and P. Huovinen, J. Phys. G 35, 104125 (2008) [arXiv:0806.1367[nucl-th]].

[6] D. Molnar, arXiv:0806.0026 [nucl-th].

[7] A. El, A. Muronga, Z. Xu and C. Greiner, arXiv:0812.2762 [hep-ph].

[8] M. Cheng et al., Phys. Rev. D 77, 014511 (2008) |arXiv:0710.0354[hep-lat]].

[9] G. Policastro, D. T. Son and A. O. Starinets, Phys. Rev. Lett. 87, 081601 (2001); P. Kovtun, D. T. Son and A. O. Starinets, Phys. Rev. Lett. 94, 111601 (2005)

[10] H. B. Meyer, Phys. Rev. Lett. 100, 162001 (2008) arXiv:0710.3717 [hep-lat]].

[11] R. J. Fries, B. Muller and A. Schafer, Phys. Rev. C 78, 034913 (2008) [arXiv:0807.4333 [nucl-th]].

[12] A. Krasnitz, Y. Nara and R. Venugopalan, Phys. Rev. Lett. 87, 192302 (2001); Y. V. Kovchegov, Nucl. Phys. A 762, 298 (2005) |arXiv:hep-ph/0503038. 